\title{
Evaluation of QT Intervals in Compensated and Decompensated Chronic Hepatitis C Cirrhotic Patients
}

\author{
Osama Ahmed Khalil ${ }^{1}$, Mohamed Samy Fawzy ${ }^{2}$, Ashraf Khalifa EL-Naggar ${ }^{1}$, and Entesar Kamel Sayed ${ }^{* 3}$ \\ ${ }^{1}$ Internal Medicine Department, Faculty of Medicine, Zagazig University, Zagazig Egypt, \\ ${ }^{2}$ Medical Biochemistry Department, Faculty of Medicine, Zagazig University, Zagazig, Egypt, \\ ${ }^{3}$ Internal Medicine Department, Ahmed Maher Teaching Hospital, Egypt \\ *Corresponding authors: Entesar Kamel Sayed Sayed, Mobile: (+20)1007397091, Email: drayaahmed@ yahoo.com
}

\begin{abstract}
Background: Cirrhotic cardiomyopathy is chronic cardiac dysfunction in cirrhotic patients with no previous structural heart disease. QT prolongation is one of the most important cardiac alterations related to cirrhosis.

Objective: This study was aimed to evaluate QT interval duration among compensated and decompensated chronic Hepatitis C (Ch HCV) cirrhotic patients and to detect its relation to biochemical changes in cirrhosis and its effect on patients' outcome. Patients and Methods: A cross sectional study was conducted on $52 \mathrm{Ch} \mathrm{HCV}$ patients divided into two groups according to presence of signs of decompensation. Patients underwent clinical, laboratory and electrocardiographic evaluation. Cirrhosis severity was classified according to the Child-Pugh score. The QT interval in lead II (QTII), maximum QT (QTmax), heart rate corrected QTmax (msillisecond) obtained manually using by a 12-lead electrocardiogram. Results: Decompensated patients had non-significantly lower values of QTII and QT max. There was significant difference between both groups regarding QT maxc. $80.8 \%$ versus $38.5 \%$ of decompensated and compensated patients had prolonged QTC. There was significant relation between child class and QT parameters. There was significant relation between QT maxc prolongation and serum Albumin, sodium, ammonia, Urea, AFP and APACHE-II. There was significant relation between outcome and both QT max and QT maxc. Cutoff of QT max n predicting mortality was $\geq 435$ with sensitivity $80 \%$ and specificity $69 \%$. QT maxc cutoff $\geq 508$ predicted mortality with sensitivity $80 \%$ and specificity $84 \%$.
\end{abstract}

Conclusion: A prolonged QT maxc was prevalent among cirrhotic patients and this positively correlated with disease severity and high mortality.

Keywords: Cirrhosis, prolongation, cardiomyopathy.

\section{INTRODUCTION}

Liver cirrhosis is a progressive pathological process characterized by fibrosis and nodular regeneration. A variety of well-known etiologies can cause cirrhosis, such as hepatitis virus infection (hepatitis B and C virus), drugs (including alcohol), autoimmune diseases, genetic diseases and nonalcoholic steatohepatitis. In addition to liver damage, cirrhotic patients have renal, pulmonary, hemodynamic and cardiac dysfunction that increase morbidity and mortality ${ }^{(\mathbf{1})}$.

Cirrhotic cardiomyopathy has been used to describe chronic cardiac dysfunction in cirrhotic patients with no previous structural heart disease ${ }^{(\mathbf{1})}$. It is defined by the finding of one or more of the following changes: normal or augmented systolic function at rest but poor contractile response to stress; diastolic dysfunction; structural abnormalities in cardiac chambers; and electrophysiological changes. These abnormalities occur to a varied degree in up to $50 \%$ of cirrhotic patients ${ }^{(2-3)}$. Most patients with cirrhotic cardiomyopathy are asymptomatic and, for this reason, complementary exams are important to identify them. The electrocardiography (ECG) is a low-cost and noninvasive method that may help to identify patients with cirrhotic cardiomyopathy ${ }^{(4)}$.

QT prolongation is one of the most important cardiac alterations related to cirrhosis and is easily

determined by ECG. A prolonged QT interval is associated with a higher mortality rate among patients with chronic liver disease ${ }^{(5)}$. As it is associated with ventricular arrhythmias as well as sudden cardiac death in both congenital and acquired conditions ${ }^{(6)}$.

Most of previous studies have shown that end stage liver disease (ESLD) is associated with several electrophysiological changes; specifically, increased prevalence of QT prolongation ${ }^{(7)}$. While the exact mechanism for QT prolongation is still unknown, both improvement in liver function and liver transplantation have been associated with significant shortening in the QT interval in studies with small sample sizes ${ }^{(3)}$.

This study was aimed to find out frequency of prolongation of QT interval corrected to heart rate among chronic hepatitis $\mathrm{C}$ cirrhotic compensated and decompensated patients, to test the effect of prolongation of QTc interval on short-term patient outcome and to find most important predictors of prolonged QTc interval in those patients.

\section{PATIENT AND METHODS}

This Prospective cohort cross sectional study included a total of $52 \mathrm{Ch} \mathrm{HCV}$ patients, attending at Internal Medicine department of Zagazig University hospitals and Ahmed Maher teaching hospital. Written informed consent of all the subjects was obtained. This study was conducted between November 2018 to June 2019.

Ethical approval:

Approval for doing the research was obtained from Internal Medicine and Medical Biochemistry 
Departments, Faculty of Medicine, Zagazig 2 University after receiving Institutional Review Board (IRB) approval.

The included subjects were divided into two groups; Group (1) consisted of 26 compensated chronic Hepatitis C cirrhotic patients, with no sign and symptoms of decompensation (Jaundice, ascites, variceal bleeding or hepatic encephalopathy) and Group (2) consisted of 26 decompensated chronic Hepatitis C cirrhotic patients with hepatic encephalopathy, variceal bleeding, ascitic or jaundice.

\section{Inclusion criteria:}

1) Chronic Hepatitis $\mathrm{C}$ cirrhotic patients with and without treatment.

2) Age $>18$ years and either sex.

\section{Exclusion criteria:}

1) Patients having any underlying condition that may predispose the prolongation of the QT interval e.g. structural heart disease (left ventricular hypertrophy, heart failure, myocardial ischemia), alcohol toxicity, hypertension, electrolyte abnormalities i.e. (hypokalemia, hypocalcemia and hypomagnesemia).

2) Patients taking medication known to affect QT e.g. anti -arrhythmic drugs (sotalol, amiodarone), antipsychotic drugs (haloperidol, ziprasidone) and second generation of antihistamines (astemizole), beta blocker e.g., (propranolol)

3) Patients with atrial fibrillation or flutter.

4) Patients with HCC or receiving chemotherapy.

5) Co infected patients with HBV or HIV

Study Assessments and Data Collection: For each eligible patient, the following data was reported:

Complete history and full clinical examination: This was done according to the included work sheet with special stress on the presence of predisposing factors of Qt interval prolongation (hypokalemia, hypocalcemia, hypomagnesemia) hypertension, and medication such as anti- arrhythmic, anti -psychotic, and anti histaminics, in addition to sign of decompensation if present

\section{Laboratory investigations:}

1. Complete blood picture: By automated blood counter (Mindray BC 2300).
. Kidney function tests: Including serum creatinine and serum urea by colorimetric method.

3. Liver function tests: Including serum bilirubin (total and direct), albumin, ammonia, PT, ALT and AST. Fasting plasma glucose level and HbA1c before and after HCV treatment.

4. Serum electrolytes: $\mathrm{Na}^{+}, \mathrm{K}^{+}, \mathrm{Ca}^{++}$and $\mathrm{Mg}^{++}$and serum Alpha feto - protein level.

\section{B) Routine pelvi -abdominal ultrasound:}

Real time ultrasound with abdominal probe which may reveal nodularity, surface irregularity, increased echogenicity and atrophy. with advanced cirrhosis liver appears small and multinodular, ascites, decreased Doppler flow in portal circulation.

Acute Physiology and Chronic Health Evaluation (APACHE) II score $^{(8)}$ : One of several ICU scoring systems. It is applied within 24 hours of admission of a patient to an intensive care unit (ICU): an integer score from 0 to 71 is computed based on several measurements; higher scores correspond to more severe disease and a higher risk of death.

\section{Statistical Analysis}

All data were collected, tabulated and statistically analyzed using SPSS 20.0 for windows (SPSS Inc., Chicago, IL, USA) \& MedCalc 13 for windows (MedCalc Software bvba, Ostend, Belgium). Data was tested for normal distribution using the Shapiro Walk test. Qualitative data was symbolized as frequencies and relative percentages. Chi square test $\left(\chi^{2}\right)$ was used to compute difference between qualitative variables. Quantitative data was stated as mean \pm SD (Standard deviation). Mann Whitney test was used to compare medians of two groups (for nonparametric data).One way ANOVA test was used to compare between more than two dependent groups of normally distributed variables while Kruskal Wallis test ranks test was used for non-normally distributed variables. Pearson's and Spearman correlation tests were used for correlating normal and non-parametric variables respectively. Multivariate regression analysis (stepwise method) was performed to ascertain factors associated with sclerostin levels. All statistical comparisons were two tailed with significance Level of $\mathrm{P}$-value $\leq 0.05$ is significant and $p$ $\leq 0.001$ is highly significant difference.

\section{RESULTS}

Table (1): Comparison between compensated and decompensated groups as regard demographic data.

\begin{tabular}{|c|c|c|c|c|c|c|}
\hline \multicolumn{3}{|l|}{ Parameter } & $\begin{array}{l}\text { Compensated } \\
\text { (group I) N } 26\end{array}$ & $\begin{array}{l}\text { Decompensated } \\
\text { (group II) N } 26\end{array}$ & $\mathbf{t} / \mathbf{X}^{2}$ & $\mathbf{P}$ \\
\hline \multicolumn{3}{|l|}{ Age (years) } & $48.05 \pm 5.16$ & $52.55 \pm 5.51$ & -2.919 & $0.005 *(s)$ \\
\hline \multirow[t]{2}{*}{ Sex } & Male & $\mathbf{N}$ & $15(57.7 \%)$ & $15(57,7 \%)$ & \multirow[t]{2}{*}{ |---- } & \multirow[t]{2}{*}{----- } \\
\hline & Female & $\mathbf{N}$ & $11(42,3 \%)$ & $11(42,3 \%)$ & & \\
\hline
\end{tabular}

$P$ value $<0.05$ was considered statistically significant

The current study showed that there is statistically non-significant relation between liver decompensation and gender however decompensated patients were significantly older (Table 1). 
Table (2): Comparison between compensated and decompensated groups as regard Mean value \pm SD of QTII, QTmax and QTmaxc prolongation and percentage at the time of admission.

\begin{tabular}{|c|c|c|c|c|c|c|}
\hline \multicolumn{3}{|l|}{ Parameter } & $\begin{array}{l}\text { Compensated } \\
\text { (group I )N } 26\end{array}$ & $\begin{array}{l}\text { Decompensated } \\
\text { (group II)N } 26\end{array}$ & $\mathbf{t} / \mathbf{X}^{2}$ & $\mathbf{P}$ \\
\hline \multicolumn{3}{|c|}{ QT II (mesc) } & $376.82 \pm 31.6$ & $395.55 \pm 53.49$ & -1.537 & 0.131 \\
\hline \multicolumn{3}{|c|}{ QT max (mesc) } & $398.58 \pm 33.9$ & $424.44 \pm 56.7$ & -1.996 & 0.05 \\
\hline \multicolumn{3}{|c|}{ QT maxc (mesc) } & $462.47 \pm 32.9$ & $485.94 \pm 46.79$ & -2.092 & $0.042 *$ \\
\hline \multirow{2}{*}{$\begin{array}{l}\text { Qtc- } \\
\text { Prolonged\% }\end{array}$} & No & $\mathbf{N}$ & $16(61.5 \%)$ & $5(19.2 \%)$ & \multirow{2}{*}{9.65} & \multirow{2}{*}{$0.002 *(s)$} \\
\hline & Yes & $\mathbf{N}$ & $10(38.5 \%)$ & $21(80.8 \%)$ & & \\
\hline
\end{tabular}

Regarding QT II and QT max, decompensated patients had non-significantly lower values $(372.82$, and 395.55 in compensated versus 395.55, and 424.44 in decompensated for QT II and QT max respectively. However there is statistically significant difference between both groups regarding QT maxc where it was longer in decompensated patients $(485.94 \pm 46.79)$ with $80.8 \%$ of them had prolonged QTC versus 0nly $38.5 \%$ in compensated patients (table 2)

Table (3): Relation between QTII, QTmax and QTmaxc and child-pough classification among the studied patients.

\begin{tabular}{|l|l|l|l|l|l|}
\hline & Child A & Child B & Child C & F & P -value \\
\hline QT II(mesc) & $372.76 \pm 29.9$ & $390.0 \pm 35.4$ & $395.5 \pm 53.9$ & 1.823 & 0.172 \\
\hline QTmax (mesc) & $376.61 \pm 34.5$ & $410.0 \pm 33.3 \mathbf{a}$ & $434.4 \pm 56.79$ ab & 3.598 & $0.032^{*}$ \\
\hline QT maxc(mesc) & $452.58 \pm 34.51$ & $487.62 \pm 17.55 \mathbf{a}$ & $494.94 \pm 46.7 \mathbf{a}$ & 3.489 & $0.034 *$ \\
\hline
\end{tabular}

F ANOVA test a significant in comparison to child A b significant in comparison to child B

There is significant relation between child class and QT parameters. On LSD comparison, patients with Child C had significantly higher QT max and B higher than A. but regarding QT maxc, patients with child C \& B had significantly higher values than those with child A while there was no significant difference in QT maxc between child B and C (Table 3).

Table (4): Univariate analysis for detection of QT maxc prolongation predictors of the studied groups.

\begin{tabular}{|c|c|c|c|c|}
\hline & $\begin{array}{l}\text { Prolonged } \\
(\mathrm{N}=31)\end{array}$ & $\begin{array}{l}\text { NOT } \\
(\mathrm{N}=21)\end{array}$ & $\begin{array}{l}\text { t/ Mann } \\
\text { Whitney }\end{array}$ & $\mathbf{P}$ \\
\hline Age (years) & $50.7 \pm 5.27$ & $48.0 \pm 5.94$ & 1.726 & 0.090 \\
\hline Hb (g/dl) & $11.41 \pm 2.43$ & $12.42 \pm 2.28$ & -1.501 & 0.140 \\
\hline TLC $\left(\times 10^{3}\right)$ & $6.43 \pm 2.41$ & $5.97 \pm 2.1$ & 0.689 & 0.494 \\
\hline PLT $\left(\times 10^{3}\right)$ & $145.22 \pm 71.6$ & $164.71 \pm 72.3$ & -0.810 & 0.422 \\
\hline INR & $1.53 \pm 0.40$ & $1.29 \pm 0.46$ & 1.924 & 0.060 \\
\hline $\mathrm{Na}^{+}(\mathbf{m E q} / \mathbf{L})$ & $133.96 \pm 6.15$ & $137.14 \pm 3.45$ & -2.143 & $0.037 *$ \\
\hline $\mathrm{K}^{+}(\mathbf{m E q} / \mathbf{L})$ & $4.09 \pm 0.42$ & $4.05 \pm 0.41$ & 0.330 & 0.743 \\
\hline $\mathrm{Ca}^{++}(\mathrm{mg} / \mathrm{dL})$ & $8.99 \pm 0.52$ & $9.21 \pm 0.5$ & -1.516 & 0.136 \\
\hline $\mathrm{Mg}^{++}(\mathrm{mg} / \mathrm{dL})$ & $2.08 \pm 0.22$ & $2.07 \pm 0.17$ & 0.104 & 0.917 \\
\hline Ammonia ( $\mu \mathrm{g} / \mathrm{dL})$ & $116.74 \pm 25.85$ & $96.71 \pm 25.1$ & 2.773 & 0.008* \\
\hline Total Bil (mg/dL) & $1.83 \pm 0.87$ & $1.52 \pm 1.18$ & 1.060 & 0.294 \\
\hline Direct Bil (mg/dL) & $0.92 \pm 0.78$ & $0.69 \pm 0.7$ & 1.120 & 0.268 \\
\hline Albumin (g/dL) & $2.95 \pm 0.93$ & $3.77 \pm 0.71$ & -3.407 & $0.001 * *$ \\
\hline Urea (mg/dL) & $75.59 \pm 34.2$ & $33.66 \pm 14.6$ & 2.474 & 0.017* \\
\hline Creatinine (mg/dL) & $1.32 \pm 0.33$ & $1.15 \pm 0.35$ & $-\mathbf{0 . 1 0 7}$ & 0.916 \\
\hline AFP (ng/mL) & $6.55 \pm 4.93$ & $2.08 \pm 0.94$ & 3.177 & $0.003 *$ \\
\hline APACHE_II & $18.19 \pm 11.0$ & $12.9 \pm 2.9$ & 2.145 & $0.037 *$ \\
\hline
\end{tabular}

There is statistically significant relation between QT maxc prolongation and all of serum Albumin (lower in prolonged QT maxc), Na, ammonia, Urea, AFP and APACHE-II (higher with prolonged QT maxc). On the other hand, there was non-significant relation between QT prolongation and any of demographic or other laboratory parameters (Table 4). 
Table (5): Multivariate logistic regression for independent predictors for QT maxc prolongation of the studied groups.

\begin{tabular}{|c|c|c|c|c|c|}
\hline \multirow{2}{*}{ Parameter } & \multirow{2}{*}{ Wald } & \multirow{2}{*}{$\mathbf{P}$} & \multirow{2}{*}{ OR } & \multicolumn{2}{|c|}{ 95\% C.I. for } \\
\hline & & & & Lower & Upper \\
\hline $\mathrm{Na}^{+}(\mathbf{m E q} / \mathrm{L})$ & 0.117 & 0.732 & 0.967 & .800 & 1.169 \\
\hline Ammonia $(\mu \mathrm{g} / \mathrm{dL})$ & 0.323 & 0.570 & 1.009 & .977 & 1.043 \\
\hline Albumin (g/dL) & 0.025 & 0.875 & 1.102 & .329 & 3.694 \\
\hline Urea (mg/dL) & 1.353 & 0.245 & 1.013 & .991 & 1.036 \\
\hline $\operatorname{AFP}(\mathbf{n g} / \mathbf{m l})$ & 2.821 & 0.093 & 1.209 & .969 & 1.509 \\
\hline APACHE_II & 0.763 & 0.382 & 1.059 & .931 & 1.206 \\
\hline
\end{tabular}

On doing logistic regression for variables significantly associated with prolonged QT maxc, higher ammonia, urea, alpha fetoprotein and APACHE II score non-significantly increased such risk (Table 5)

Table (6): Relation QTII, QTmax and QTmaxc and patient outcome.

\begin{tabular}{|l|l|l|l|l|}
\hline & $\begin{array}{l}\text { Died } \\
(\mathbf{N}=5)\end{array}$ & $\begin{array}{l}\text { Survived } \\
(\mathrm{N}=47)\end{array}$ & $T$ & $P$-value \\
\hline QTII (mesc) & $\mathbf{4 3 0 . 0} \pm \mathbf{6 4 . 0 3}$ & $\mathbf{3 7 8 . 3 4} \pm 35.32$ & 1.859 & $\mathbf{0 . 0 5 9}$ \\
\hline QTmax (mesc) & $\mathbf{4 7 2 . 0} \pm 65.72$ & $400.68 \pm 36.27$ & 3.844 & $\mathbf{0 . 0 0 * *}$ \\
\hline QTmaxc (mesc) & $\mathbf{5 2 6 . 6 8} \pm \mathbf{1 7 . 3 3}$ & $\mathbf{4 6 8 . 5 9} \pm 36.03$ & 3.537 & $\mathbf{0 . 0 0 1} * *$ \\
\hline
\end{tabular}

$\mathrm{t}$ test for statistical difference between quantitative independent two parameters.

On follow up of studied patients, 9.6\% had died by the end of the study (Figure 1). On assessing relation between mortality and QT, there was significant relation between it and both QT max and QT maxc (shorter in survivors) while non-significant relation was found between mortality and QT II values (Table 6)

Table (7): ROC Curve for detection cutoffs for mortality regarding QTmax, QTmaxc and APACHE II score.

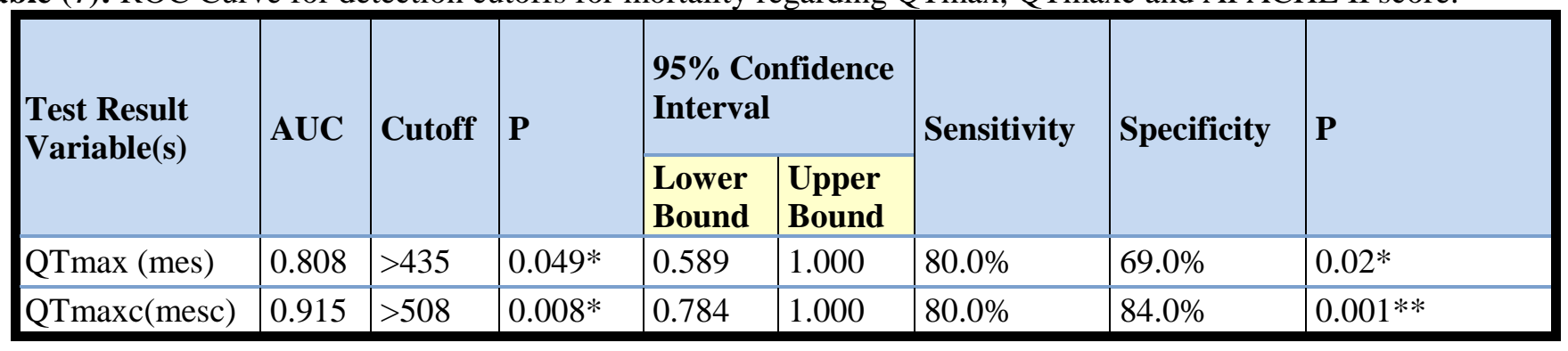

\section{AUC area under curve}

On assessing performance of QT levels in predicting mortality, best cutoff of QT max cutoff in predicting mortality was $\geq 435$ with area under curve 0.808 , and sensitivity $80 \%$ and specificity $69 \%$. The best QT maxc cutoff for predicting mortality was $\geq 508$ with area under curve 0.915 , sensitivity $80 \%$ and specificity $84 \%$ (Table 7 ). 


\section{DISCUSSION}

Most of the previous studies have shown that end stage liver disease (ESLD) is associated with several electrophysiological changes; specifically, increased prevalence of QT prolongation ${ }^{(7)}$. On the other hand, other study documented no QT changes in $\mathrm{LC}^{\left({ }^{(9)} \text {. }\right.}$

Multiple factors are thought to be responsible for QT prolongation including electrolyte abnormalities, myocardial ischemia, medications, alcohol toxicity, and autonomic imbalance with sympathetic nervous system hyperactivity ${ }^{(10)}$.

While the exact mechanism for QT prolongation is still unknown, both improvement in liver function and liver transplantation have been associated with significant shortening in the QT interval (3). Likewise, previous studies demonstrate reduction in the QT interval for cirrhotic patients who receive beta-adrenergic blockade in both the acute and chronic settings ${ }^{(\mathbf{1 1})}$.

In the current study, QTII, QT max and QT maxc of the studied groups at the time of admission was assessed. QT maxc was prolonged to more than (450 msec) in 31 patients with prevalence $59.6 \%$, and this was in agreement with the study of Tsiompanidis et al. ${ }^{(12)}$ who found that QT and QTC intervals were substantially prolonged in patients with cirrhosis and these prolongation is not related to the etiology of cirrhosis. Fouda et al. ${ }^{(\mathbf{1 3})}$ where also found that around 53\% of the studied hospitalized Hepatitis C positive non -alcoholic liver cirrhosis patients had prolonged heart rate corrected QT. (QT maxc). Bal et al. ${ }^{(14)}$ and Li et al. ${ }^{(15)}$ also found that the prevalence of QTC prolongation was $46.93 \%$ and $56 \%$ respectively, However these finding was in contrast with those obtained by Hansen et al. ${ }^{(9)}$ who found no difference in QT between patients with cirrhosis and controls. This difference in those studies might be due to nonexclusion of their patients on beta blocker and they measure the dispersion QT not QTmaxc in the previous studies.

One of the mechanisms suggested to play an important role in the pathogenesis of QT prolongation in patients with cirrhosis, is the enhanced sympathetic nervous system activity ${ }^{(16)}$ and increased circulating levels of noradrenalin, and it is an index of enhanced sympatho- adrenal activity, observed in patients with advanced liver disease ${ }^{(\mathbf{1 7})}$.

Also, our study found that decompensated patients (group II) were significantly higher in QTII, QT max and QT maxc than compensated patients (group1) with mean value \pm SD (485.94 \pm 46.79) $(\mathrm{p}<0.05)$ vs $(462.47 \pm 32.9)$.this was consistent with Tsiompanidis et al. ${ }^{(12)}$, who found that patients with decompensated cirrhosis, in comparison with those with compensated cirrhosis, had longer QTc (435.3 \pm $30.4 \mathrm{msec} v s 419.0 \pm 30.1 \mathrm{msec}, P=0.070)$ and they had severe Cardiac autonomic nervous activity than those with compensated cirrhosis, while they did not found significant differences in QTII $(P=0.55)$ between cirrhotic patients and controls.

Tsiompanidis et al. ${ }^{(23)}$ implies that when liver disease progresses to a point where the human body cannot overcome the cirrhosis effect, one of the clinical features of this process is the exacerbation of the cardiac electrical conductance abnormalities.

On assessing the relationship between QT intervals prolongation and the severity of the disease as measured by Child-Pugh score, child C and child B chronic hepatitis $\mathrm{C}$ cirrhotic patients had a significant prolongation of QT max and QTmaxc than child A cirrhotic patients. This was consistent with Lanzieri et al. ${ }^{(18)}$ who showed a longer QT interval in Child-Pugh $\mathrm{C}$ cirrhotic patients and also there was a positive correlation between QT interval and the Child-Pugh score in individuals with Child-Pugh scores $>7(\mathrm{r}=0.05, \mathrm{p}<0.05)$ as well as Mimidis et al. ${ }^{(19)}$ and Mozos et al. ${ }^{(20)}$.

Also, in large study on 409 cirrhotic patients done by Bal $\boldsymbol{e t}$ al. ${ }^{(14)}$, they reported higher corrected QT in the Child-Pugh C group than child B group $(451 \pm 43 \mathrm{vs} 434 \pm 31 \mathrm{~ms}, \mathrm{p}<0.001)$ despite similar age and gender.

Genovesi et al. ${ }^{(21)}$ demonstrated a significant correlation between the stage of cirrhosis according to the Child-Pugh classification, the hepatic venous pressure gradient (HVPG) and QT interval prolongation, which was more common in patients with alcoholic than viral cirrhosis.

These findings were in contrast with Carey and Douglas ${ }^{(22)}$, Adigun et al. ${ }^{(7)}$, Fouda et al. ${ }^{(13)}$, and Tsiompanidis et al. ${ }^{(\mathbf{1 2})}$ as they reported that QT maxc did not show significant correlation to Child-Pugh score

The importance of normal liver functions on preservation of the electrophysiological properties of the heart is supported by several studies that have examined the prolongation of QTc before and after liver transplantation. Data suggest that liver transplantation improved the prolonged QTc, however the extent and the degree of the improvement was variable ${ }^{(23)}$.

In the current study there was a significant negative correlation between QTII, QT max and QT maxc and each of serum albumin $(\mathrm{p}=0.001)$ and serum sodium. This was consistent with Cheng et al. ${ }^{(25)}$ who found that patients with abnormal QTC interval had lower serum albumin levels than those with normal QTC and there was a significant negative association

Bahrami et al. ${ }^{\text {(26) }}$ reported that a low albumin level is associated with inflammation, and impaired synthetic function of liver and this has been linked to diastolic dysfunction in humans which indicate the association between inflammation and ventricular arrhythmias. These findings were in contrast with Pourafkari $\boldsymbol{e t}$ al. ${ }^{(27)}$ who found that serum creatinine is the only independent factor that significantly 


\section{ejhm.journals.ekb.eg}

correlate with QT interval, and patients with prolonged QTC interval had a significantly lower serum creatinine than patients with normal QTC interval $(\mathrm{p}=0.013)$.

Genovesi et al. ${ }^{(21)}$ noted that QTC interval was inversely correlated with plasma calcium ion concentration. In our study patients with electrolytes abnormalities were excluded, this may suggest the contribution of other intrinsic cardiac factor rather than electrolyte disturbance in the pathogenesis of cardiac abnormalities.

Our study did not find a significant correlation between QTmaxc prolongation with each of serum K, $\mathrm{Ca}$ and $\mathrm{Mg}$ ( $\mathrm{p}=0.743,0.136,0.917$ respectively). This was consistent with Pourafkari et al. ${ }^{(27)}$ and Cheng et al. ${ }^{(25)}$ who found no significant difference between the two groups in terms of other finding including serum electrolytes $(p>0.05)$ for all variables.

In the current study, there was a significant positive correlation between QTII, QT max, QTmaxc with serum ammonia and alpha fetoprotein while nonsignificant correlation was found between QT with serum bilirubin and creatinine. This was consistent with Negru et al. ${ }^{(28)}$ who detected non-significant correlation between biochemical parameters tested and corrected QTC. They conveyed that QT $\max$ significantly positive correlated with total protein $(r=0.402, p<0.01)$, total and direct bilirubin. $(r=0.366, p<0.05 ; r=0.332, p<0.05)$ and significantly negative correlated with serum triglyceride $(r=0.357$, $\mathrm{p}<0.05)$ while QTC significantly correlated with only total protein and triglycerides $(r=0.308, p<0.05$; $\mathrm{r}=0.344, \mathrm{p}<0.05)$ but not with uric acid level and no other parameters tested was correlated with QTmaxc.

Zhao et al. ${ }^{(29)}$ reported, in their entire cohort, that there was a significant correlation between QTC prolongation and each of serum bilirubin level, blood urea nitrogen, serum creatinine, prothrombin time, international normalized ratio, Child-Pugh score ,lower red blood cell (RBC), hemoglobin (HB), serum albumin (ALB), alanine aminotransferase and serum calcium, however the limited number of decompensated patients in our study might explain this difference.

There was a significant positive correlation between QTmaxc prolongation with APACHEII score. $(\mathrm{p}=0.00)$ as well as the mean APACH II score in prolonged QTmaxc patients was significantly higher than those with non-prolonged QTmaxc patients $(\mathrm{p}<0.037)$ this finding was consistent with George $\boldsymbol{e} t$ al. ${ }^{(30)}$, in considering the prolongation of QTmaxc as a marker of increased prediction of mortality with high APACHE score.

After 3 months follow up for prolonged QTmaxc patients, we found that $9.6 \%$ had died with QT max cutoff $>435$ and QTC cut off $>508$ can predict mortality. Kim et al. ${ }^{(24)}$ who reported that, in their study, (46.1\%) died confirming the high mortality in ESLD and this high mortality was seen at all level of prolonged QT.

George et al. ${ }^{(30)}$ found that patients with a prolonged QTC had three times the odds of an adverse outcome in the ICU than people with normal QTC.

This represented adverse outcome associated with QTC prolongation. Bal et al. ${ }^{(\mathbf{1 4})}$ did not find an independent effect of QTC interval in mortality in their cox regression analysis, adjusted for the Child-Pugh score .they studied corrected QTC as regard to the Child-Pugh score only and did not study other factors associated with cirrhosis and may be related to QTC prolongation associated mortality. QT prolongation has been associated with increased mortality secondary to ventricular arrhythmias and sudden cardiac death.

The strength of the current study is that we examined subjects under controlled conditions and the potential confounding effects of medication, food intake and coffee consumption have been avoided. Regarding to medication, recent data suggested that propranolol administration reduce QT interval in patients with advanced liver cirrhosis waiting for liver transplantation ${ }^{(11)}$. Thus, discontinuation of beta blocker from our patients eliminated the effect of this medication on QT interval duration and allowed us to examine the net effect of the disease on QT interval duration

The study had some limitations. The number of the participants was not large and we did not examine for the presence of cirrhotic cardiomyopathy to look for associations between QT-related parameters and indices of systolic or diastolic function of the heart by echo which is recommended to be done in further study. Lack of control group, and short follow up period were among study limitations.

\section{CONCLUSION}

It could be concluded that prolongation of QT intervals was found in chronic Hepatitis C cirrhotic patients and this prolongation is increased in decompensated LC and patients with child B and C cirrhosis. Such prolongation is associated with high mortality rate in those patients.

\section{Funding: None to declare}

Conflict of Interest: None to declare

\section{REFERENCES}

1. Gassanov N, Caglayan E, Semmo N et al. (2014): Cirrhotic cardiomyopathy: a cardiologist's perspective. World J Gastroenterol., 20(42): 15492-8.

2. Zamirian M, Tavassoli M, Aghasadeghi K (2012): Corrected QT interval and QT dispersion in cirrhotic patients before and after liver transplantation. Arch Iran Med., 15:375-377.

3. Rahman S, Mallett SV (2015): Cirrhotic cardiomyopathy: implications for the perioperative 
management of liver transplant patients. World J Hepatol., 7(3):507-20.

4. Mozos I (2015): Arrhythmia risk in liver cirrhosis. World J Hepatol., 7(4):662-72.

5. Bari K, Garcia-Tsao G (2012): Treatment of portal hypertension. World J Gastroenterol., 18(11):1166-75.

6. Van Noord C, Eijgelsheim M, Stricker BH (2010): Drug- and non-drug-associated QT interval prolongation. British Journal of Clinical Pharmacology, 70(1): 16-23.

7. Adigun AQ, Pinto AG, Flockhart DA et al. (2005): Effect of cirrhosis and liver transplantation on the gender difference in QT interval. Am J Cardiol., 95:691-694.

8. Knaus WA, Draper EA, Wagner DP (1985): APACHE II: a severity of disease classification system. Crit Care Med., 13(10):818-29.

9. Hansen S, Møller S, Bendtsen F et al. (2007): Diurnal variation and dispersion in QT interval in cirrhosis: relation to hemodynamic changes. J Hepatol., 47:373380.

10. Tarapués M, Cereza G, Arellano AL et al. (2014): Serious QT interval prolongation with ranolazine and amiodarone. Int J Cardiol., 172:60-61.

11. Kim YK, Hwang GS, Shin WJ et al. (2011): Effect of propranolol on the relationship between QT interval and vagal modulation of heart rate variability in cirrhotic patients awaiting liver transplantation. Transplant Proc., 43:1654-1659.

12. Tsiompanidis E, Siakavellas SI, Tentolouris A et al. (2018): Liver cirrhosis-effect on QT interval and cardiac autonomic nervous system activity. World Journal of Gastrointestinal Pathophysiology, 9(1): 28-36.

13. Fouda R, Elghobary $M$ (2012): QT Intervals in Hospitalized Hepatitis C Positive Non Alcoholic Liver Cirrhosis Patients. Med J Cairo Univ., 80(2): 105-109.

14. Bal JS, Thuluvath PJ (2003): Prolongation of QTc interval: relationship with etiology and severity of liver disease, mortality and liver transplantation. Liver Int., 23 (4):243-248.

15. Li L, Liu HR, Shu JL et al. (2007): Clinical Investigation of Q-t prolongation in Hepatic Cirrhosis. Zhonghua Yi Xue Za., 87: 2717-2718.

16. Puthumana L, Chaudhry V, Thuluvath PJ et al. (2001): Prolonged QTc interval and its relationship to autonomic cardiovascular reflexes in patients with cirrhosis. J Hepatol., 35(6):733-8.

17. Bernardi M, Calandra S, Colantoni A et al. (1998): Q$\mathrm{T}$ interval prolongation in cirrhosis: prevalence, relationship with severity, and etiology of the disease and possible pathogenetic factors. Hepatology, 27(1):28-34.

18. Lanzieri PG, Gismondi RA, Chimelli MC et al. (2017): Cirrhotic Patients with Child-Pugh C Have Longer QT
Intervals: International Journal of Cardiovascular Sciences, 30(6):496-503.

19. Mimidis K, Papadopoulos V, Thomopoulos $K$ et al. (2003): Prolongation of the QTc interval in patients with cirrhosis. Ann Gastroenterol., 16: 155-158.

20. Mozos I, Costea C, Serban C et al. (2011): Factors associated with a prolonged QT interval in liver cirrhosis patients. J Electrocardiol., 44: 105-108.

21. Genovesi S, Prata Pizzala M, Pozzi M et al. (2009): QT interval prolongation and decreased heart rate variability in cirrhotic patients: relevance of hepatic venous pressure gradient and serum calcium. Clin Sci., 116: 851-859.

22. Carey EJ, Douglas DD (2005): Effects of orthotopic liver transplantation on the corrected QT interval in patients with end-stage liver disease. Dig Dis Sci., 50:320-323.

23. Liu H, Jayakumar S, Traboulsi M et al. (2017): Cirrhotic cardiomyopathy: Implications for liver transplantation, Liver Transpl., 23(6):826-835.

24. Kim SM, George B, Alcivar-Franco D et al. (2017): QT prolongation is associated with increased mortality in end stage liver disease. World Journal of Cardiology, 9(4): 347-354.

25. Cheng-Ching W, Yung-Chuan L, Teng-Hung $Y$ et al. (2018): Serum albumin level and abnormal corrected QT interval in patients with coronary artery disease and chronic kidney disease. Internal medicine Journal, 48(10):1242-1251.

26. Bahrami H, Bluemke DA, Kronmal $\mathrm{R}$ et al. (2008): Novel metabolic risk factors for incident heart failure and their relationship with obesity. Jam Coll Cardiol., 51: 1775-83.

27. Pourafkari L, Ghaffari S, Nazeri L et al. (2017): Electrocardiographic finding in hepatic cirrhosis and their association with severity of disease. Cor et Vasa., 59(2): 105-113.

28. Negru RD, Cojocaru DC, Felea M et al. (2017): QT interval parameters and ventricular arrhythmic event in liver cirrhosis-correlation with severity and etiology. In: Biomedical Research, 28(3):1130-1134.

29. Zhao J, Qi X, Hou F et al. (2016): Prevalence, Risk Factors and In-hospital Outcomes of QTc Interval Prolongation in Liver Cirrhosis. Am J Med Sci., 352:285-295.

30. George TK, Chase D, Thomas VV et al. (2015): Association between prolonged corrected QT interval and outcomes in patients in a medical Intensive Care unit. Indian J Crit Care Med., 19(6): 326-332. 